\title{
ADMISSIBILITY AND NONLINEAR VOLTERRA INTEGRAL EQUATIONS
}

\author{
R. K. MILLER ${ }^{1}$
}

Abstract. Nonlinear perturbations of linear Volterra integral equations are studied in an abstract setting which contains and generalizes some earlier results on the same problem. The perturbed problem is first written as a variation of constants equation on a Fréchet space. It is then shown that standard fixed point theorems may be applied if the linear equation is admissible w.r.t. a Banach subspace of the Fréchet space. This theory is applied to an example where $L^{2}$-stability is proved.

I. Introduction. The purpose of this paper is to study the behavior of solutions of a nonlinear system

$$
x(t)=f(t)+\int_{0}^{t} a(t, s)(x(s)+g(s, x(s))) d s
$$

given certain information concerning the corresponding linear system

$$
y(t)=f(t)+\int_{0}^{t} a(t, s) y(s) d s .
$$

These equations will be studied in the abstract form

$$
x=f+T(x+g(x))
$$

and

$$
y=f+T y
$$

where $x, y$ and $f$ are elements of a Fréchet space $\mathfrak{F}, T: \mathcal{F} \rightarrow \mathfrak{F}$ is a continuous linear map and $g: \mathfrak{F} \rightarrow \mathcal{F}$ is a nonlinear map. Let $X$ be a linear subspace of $\mathcal{F}$. Assume that $(\mathrm{L})$ is an admissible w.r.t. $(X, X)$; that is, for each $f \in X$ equation (L) admits a solution $y \in X$. The problem is to show that $(\mathrm{N})$ also has a solution $x$ in $X$.

For example, it is easy to give conditions on $f, a$ and $g$ which insure that equation (1) admits a unique continuous solution $x(t)$. It is much harder to show that $x(t)$ is bounded on the interval $R^{+}=$

Received by the editors June 2, 1969.

AMS Subject Classifications. Primary 4513, 4530; Secondary 4790, 3453.

Key Words and Phrases. Nonlinear Volterra integral equations, perturbations theory, stability theory, admissibility.

1 This research was supported in part by the National Aeronautics and Space Administration under Grant No. NGL-40-002-015 and in part by the United States Army, Durham, under Grant No. 31-124-ARO-D-270. 
$\{t: 0 \leqq t<\infty\}$. This problem may be placed in the abstract setting above if one defines $\mathcal{F}=C\left(R^{+}\right)$(with the topology of uniform convergence on compact subsets of $\left.R^{+}\right), X=B C\left(R^{+}\right)=\left\{\phi \in C\left(R^{+}\right): \phi\right.$ is bounded on $\left.R^{+}\right\}$and

$$
T \phi(t)=\int_{0}^{\iota} a(t, s) \phi(s) d s, \quad \forall \phi \in \mathcal{F} .
$$

The key assumption that $(L)$ is admissible w.r.t. $(X, X)$ means that for each $f$ in $B C\left(R^{+}\right)$the solution $y(t)$ of $(2)$ is in $B C\left(R^{+}\right)$. In other words, one has admissibility if and only if the linear system (2) is "bounded input-bounded output stable."

Admissibility has been studied by many authors. The idea seems to have originated with Massera and Schäffer [1] and has been applied to integral equations by Corduneanu [2], [3] and Antosiewicz [4]. The results in this paper are closely related to the results of Corduneanu but are more easily and more widely applicable to certain problems of the form (1).

2. Main results. Let $\mathcal{F}$ be a Fréchet space, that is $\mathcal{F}$ is both a vector space and a complete metric space with metric $\rho$ such that

(a) vector addition and scalar multiplication are $\rho$-continuous, and

(b) $\rho$ is additively invariant, i.e. $\rho(x, y)=\rho(x-y, 0)$. Let $X_{1}$ and $X_{2}$ be linear subspaces of $\mathcal{F}$ which admit norms \|\|$_{1}$ and \|\|$_{2}$. Assume

(A1) $X_{i}$ is a $B$-space under the norm \|\|$_{i}$. Moreover, \|\|$_{i}$ is stronger than the topology induced from $\mathcal{F}$ in the sense that if $\left\|x_{n}-x\right\|_{i} \rightarrow 0$ as $n \rightarrow \infty$ then $x_{n} \rightarrow x$ in $\mathcal{F}$.

(A2) $T: \mathcal{F} \rightarrow \mathcal{F}$ is a continuous linear map such that if $I=$ identity map, then $(I-T): \mathcal{F} \rightarrow \mathcal{F}$ is both one-to-one and onto.

(A3), $f \in \mathcal{F}$ and $g: \mathcal{F} \rightarrow \mathcal{F}$.

Lemma 1. If (A1-3) are satisfied then equation $(\mathrm{N})$ is equivalent to

$$
x=y-R g(x)
$$

where $R=I-(I-T)^{-1}$ is a continuous linear map of $\mathcal{F}$ into $\mathcal{F}$ and $y=f$ $-R f$ is the solution of $(\mathrm{L})$.

Proof. From the definition of $R$ it follows that $(I-R)=(I-T)^{-1}$. Thus $y=(I-T)^{-1} f=(I-R) f$ solves (L). Since $I-T$ is continuous, it is a closed map on $\mathscr{F} \times \mathcal{F}$. Thus $(I-T)^{-1}$ is closed, linear and everywhere defined on $\mathcal{F}$. By the closed graph theorem $I-R$ (and so also $R$ ) is continuous on $\mathcal{F}$. 
Subtracting $T x$ from both sides of equation (N) and applying $(I-T)^{-1}$ one obtains

$$
x=(I-T)^{-1} f+(I-T)^{-1} T g(x)=(I-R) f+(I-T)^{-1} T g(x) .
$$

Since $y=(I-R) f$ and $(I-T)^{-1} T=-R$ this reduces to equation (V). The entire calculation is reversible so that $(V)$ and $(N)$ are equivalent. Q.E.D.

Equation (V) is a "variation of constants" form of equation $(\mathrm{N})$. For the Volterra integral equation (2) the map $T$ is always a continuous map. The assumption that $I-T$ is one-to-one and onto is just the familiar theorem that linear Volterra integral equations have unique solutions. Moreover, the map $R$ will have the form

$$
R \phi(t)=\int_{0}^{t} r(t, s) \phi(s) d s,
$$

where $r(t, s)$ is the resolvent kernel, i.e. $r(t, s)$ solves the equation

$$
r(t, s)=-a(t, s)+\int_{s}^{t} a(t, u) r(u, s) d u .
$$

Once the variation of constants equation $(\mathrm{V})$ is obtained, it is easy to apply various fixed point theorems to $(\mathrm{V})$. First consider contraction maps. The next definition and lemma follow Corduneanu [2].

Definition 1. Let (A1-2) be satisfied. Then the pair $\left(X_{1}, X_{2}\right)$ is called admissible w.r.t. the map $R$ if and only if for each $f \in X_{1}, R f \in X_{2}$.

This admissibility is easily seen to be equivalent to the assumption that for each $f$ in $X_{1}$ the solution $y$ of $(I)$ is in $X_{2}$ in the special case where $X_{1}=X_{2}$.

Lemma 2. If (A1-2) hold and if $\left(X_{1}, X_{2}\right)$ is admissible w.r.t. $R$ then $R$ is continuous as a linear map of $X_{1}$ into $X_{2}$, that is

$$
\|R\|=\sup \left\{\|R f\|_{2}:\|f\|_{1}=1\right\}<\infty .
$$

Proof. Using the continuity of $R$ as a map of $\mathcal{F}$ into $\mathcal{F}$ and assumption (A1) the conclusion follows immediately by the closed graph theorem. Q.E.D.

THEOREM 1. Suppose (A1-3) are satisfied and in addition

(A4) $y \in X_{2}, g: X_{2} \rightarrow X_{1}$ and $\left(X_{1}, X_{2}\right)$ is admissible w.r.t. $R$.

(A5) There exists $\alpha>0$ and $r(0<r \leqq+\infty)$ such that if $z, w \in X_{2}$ with $\|z\|_{2},\|w\|_{2} \leqq r$ then $\|g(z)-g(w)\|_{1} \leqq \alpha\|z-w\|_{2}$. If $\alpha\|R\|<1$ and if $\|y\|_{2}+\|R\|\|g(0)\|_{1} \leqq r(1-\alpha\|R\|)$ then equation (N) has a unique solution $x \in X_{2}$ such that $\|x\|_{2} \leqq r$. 
Proof. Under these conditions it is easy to see that the right-hand side of equation $(\mathrm{N})$ defines a contraction mapping on the set $\left\{z \in X_{2}\right.$ : $\left.\|z\|_{2} \leqq r\right\}$. Q.E.D.

THEOREM 2. Let (A1-4) be satisfied and assume in addition

(A6) For each $\epsilon>0$ there exists $\delta>0$ such that if $\|z\|_{2},\|w\|_{2} \leqq \delta$ then $\|g(z)-g(w)\|_{1} \leqq \epsilon\left\|z_{1}-z_{2}\right\|_{2}$. If $g(0)=0$ then for each sufficiently small $r>0$ there exists $\eta>0$ such that if $\|y\|_{2} \leqq \eta$ then equation (N) has a unique solution $x \in X_{2}$ with $\|x\|_{2} \leqq r$.

Proof. Pick $\epsilon_{1}>0$ such that $\epsilon_{1}\|R\|<1$ and pick $\delta_{1}>0$ such that $\|g(z)-g(w)\|_{1} \leqq \epsilon_{1}\|Z-W\|_{2}$ if $\|z\|_{2},\|w\|_{2} \leqq \delta_{1}$. If $0<r \leqq \delta_{1}$ and if $\eta=r\left(1-\epsilon_{1}\|R\|\right)$ then the two inequalities

$$
\gamma\|R\| \leqq \epsilon_{1}\|R\|<1, \quad\|y\|_{2} \leqq r\left(1-\epsilon_{1}\|R\|\right)
$$

of the last theorem are satisfied. Q.E.D.

If $X_{1}=X_{2}$, Theorem 2 implies the following result.

Corollary 1. Let (A1-4) and (A6) be satisfied. If $g(0)=0$ then for each sufficiently small $r>0$ there exists $\eta>0$ such that if $f \in X_{1}$ and $\|f\|_{1} \leqq \eta$ then equation $(\mathrm{N})$ has a unique solution $x \in X_{2}$ with $\|x\|_{1} \leqq r$.

Proof. Since $y=(I-R) f,\|y\|_{1} \leqq(1+\|R\|)\|f\|_{1}$. Thus $\|y\|_{1}$ is small if $\|f\|_{1}$ is small. Q.E.D.

As an application of Theorem 2 and Corollary 1 we note that all of the perturbation results in [5] and [6] are special cases. The various assumptions on the resolvent $r(t, s)$ are simply conditions which insure admissibility.

3. The Schauder theorem. A convex Fréchet space is a Fréchet space $\mathcal{F}$ such that every neighborhood of the origin contains a convex subneighborhood. The only property of a convex Fréchet space which will be needed here is that the Schauder Fixed Point Theorem is true in such spaces.

THEOREM 3. (A1-4) are satisfied, $\mathcal{F}$ is a convex Frechet space and the map $R: \mathcal{F} \rightarrow \mathcal{F}$ is a compact operator. For any $r>0$ define $S_{i}(v)=\left\{x \in X_{i}\right.$ : $\left.\|x\|_{i} \leqq r\right\}$ and let $\bar{S}_{i}(r)$ be the $\mathcal{F}$-closure of $S_{i}(r)$. Suppose for some positive numbers $r$ and $s, g: \bar{S}_{2}(r) \rightarrow S_{1}(s)$ F-continuously. If $y \in X_{2}$ and if $\|y\|_{2}+\|R\| s \leqq r$ then equation $(\mathrm{N})$ has at least one solution $x \in X_{2}$ with $\|x\|_{2} \leqq r$.

Proof. For any $\phi$ in $\bar{S}_{s}(r)$ define $M \phi=y-R g(\phi)$. The assumptions easily imply that $M: \bar{S}_{2}(r) \rightarrow S_{2}(r) \subset \bar{S}_{2}(r)$ with $M$ F-continuous. It remains to show that $M\left(\bar{S}_{2}(r)\right)$ is precompact in $\mathcal{F}$. 
First note that the set $W=\left\{g(\phi): \phi \in \bar{S}_{2}(r)\right\}$ is $F$-bounded. Indeed, let $U$ be any $\mathcal{F}$-neighborhood of the origin. Since \|\|$_{1}$ is stronger than the $\mathcal{F}$-topology there exists a number $\delta>0$ such that if $\|\phi\|_{1} \leqq \delta$ then $\phi \in U$. For any $\alpha$ with $|\alpha| \leqq \delta / s$ if $\phi \in W \subset S_{1}(s)$ then $\|\alpha \phi\|_{1}$ $=|\alpha|\|\phi\|_{1} \leqq \alpha s \leqq \delta$. Therefore, $\alpha W \subset U$ if $|\alpha| \leqq \delta / s$, i.e. $W$ is $\mathcal{F}$ bounded.

Since $W$ is bounded and $R: \mathcal{F} \rightarrow \mathcal{F}$ is compact, $R(W)=M\left(\bar{S}_{2}(r)\right)$ is precompact. By Schauder's theorem $M$ has a fixed point $x \in \bar{S}_{2}(r)$. Since $x=M x$ and $M: \bar{S}_{2}(r) \rightarrow S_{2}(r)$ it follows that $x \in S_{2}(r)$. Q.E.D.

Notice that if $R$ is the integral operator (4) then rather weak assumptions on $a(t, s)$ easily imply the compactness of $R$ on $\mathfrak{F}=C\left(R^{+}\right)$. At the same time for most subspaces $X_{i}$ the compactness of $R$ as a map of $X_{1}$ into $X_{2}$ is usually very difficult to prove (and is of ten false). Thus Theorem 3 seems to be a very natural and convenient application of Schauder's theorem for Volterra equations. Theorem 3 is motivated by and is closely related to Theorem 2 of Corduneanu [2].

As an application of Theorem 3 we shall give a generalization of an $L^{2}$-stability theorem of the type studied by Sandberg [7] and Zames [8]. Consider a system on $n$ equations of the form

$$
x(t)=f(t)+\int_{0}^{t} a(t-s) g(s, x(s)) d s \quad(t \geqq 0) .
$$

Concerning (5) we assume

(K1) $g(t, x)$ is measurable in $(t, x)$ for $t \geqq 0$ and all $x$ and $g(t, x)$ is continuous in $x$ for each fixed $t$.

(K2) There exists $\gamma>0$ and a nonsingular, constant, $n$ by $n$ matrix $A$ such that $\left|A^{-1} g(t, x)-x\right| \leqq \gamma|x|$ for all $(t, x)$.

(K3) $a(t)$ is $L^{1}\left(R^{+}\right)$and the determinant $\operatorname{det}\left(I-a^{*}(s) A\right) \neq 0$ for Re $s \geqq 0$. Here $I=$ identity matrix and ${ }^{*}$ denotes the Laplace transform.

(K4) $f$ is in $L^{2}\left(R^{+}\right)$.

For any matrix $W$ let

$$
\Lambda(W)=\max \left\{|\lambda|{ }^{1 / 2}: \lambda \text { is an eigenvalue of } W^{*} W\right\}
$$

be the spectral norm of $W$. Define

$$
\alpha=\sup \left\{\Lambda\left(\left\{I-a^{*}(i w) A\right\}^{-1} a^{*}(i w) A\right):-\infty<w<\infty\right\} .
$$

THEOREM 4. If (5) satisfies (K1-4) and if $\gamma \alpha<1$ then equation (5) has a solution $x(t) \in L^{2}\left(R^{+}\right)$with $\|x\|_{L^{2}} \leqq(1+\alpha)(1-\alpha \gamma)^{-1}\|f\|_{L^{2}}$.

Proof. Let $\mathcal{F}$ be the space of locally $L^{2}$ functions on $R^{+}$with the topology of $L^{2}$ convergence on compact subsets of $R^{+}$. Let $X=X_{1}$ 
$=X_{2}=L^{2}\left(R^{+}\right)$. Equation (3) may be written abstractly as

$$
x=f+T\left[A^{-1} h(x)\right]=f+T\left[x+\left\{A^{-1} h(x)-x\right\}\right]
$$

where $T \phi(t)=\int^{t} a(t-s) A \phi(s) d s$. Assumption (K3) implies that $a(t-s) A$ has a resolvent $r(t-s)$ of class $L^{1}\left(R^{+}\right)$, cf. Paley and Wiener $\left[9\right.$, p. 60]. Thus the pair $\left(L^{2}\left(R^{+}\right), L^{2}\left(R^{+}\right)\right)$is admissible w.r.t. the operator $R$ where

$$
R \phi(t)=\int_{0}^{t} r(t-s) \phi(s) d s \quad(t \geqq 0) .
$$

The Parseval equation and the convolution theorem for $L^{2}$ Fourier transforms imply $\|R\| \leqq \alpha$.

In Theorem 3 let $s=\gamma, \quad r=(1+\alpha)\|f\|(1-\alpha \gamma)^{-1}$ and let $g(\phi)$ $=A^{-1} h(\phi)-\phi$. Clearly $\bar{S}_{2}(r)=S_{2}(r)$ and if $\phi \in S_{2}(r)$ then $g(\phi) \in L^{2}\left(R^{+}\right)$ with $\|g(\phi)\| \leqq \gamma\|\phi\|$. By Theorem 3 equation (5) has a solution $x \in L^{2}\left(R^{+}\right)$with $\|x\| \leqq r$. Q.E.D.

4. Extensions and comparisons. Corduneanu [3] studied the nonlinear equation

$$
x=f+T x+h(x)
$$

on the Fréchet space $\mathfrak{F}=C\left(R^{+}\right)$. Here $h: \mathfrak{F} \rightarrow \mathcal{F}$ is a nonlinear functional. If the pair $\left(X_{2}, X_{1}\right)$ is admissible w.r.t. the map $T$, then equation (N) above may be treated using his methods by setting $h(x)=T g(x)$. The problem with this approach is that in many interesting applications the pair $\left(X_{2}, X_{1}\right)$ is not admissible w.r.t. the map $T$.

Both points of view may be combined by studying nonlinear equations of the form

$$
x=f+T(x+g(x))+h(x)
$$

where $(A 1-3)$ are true and $g: \mathfrak{F} \rightarrow \mathfrak{F}$. The variation of constants formula $(\mathrm{V})$ implies that $(7)$ is equivalent to

$$
x=(I-R) f+(I-R) h(x)-R g(x) .
$$

Various conditions may be given to insure that (8) has a solution in $X_{2}$. For example, the method of proof of Theorem 2 is easily applied in order to prove the following theorem.

Theorem 5. Let (H1-3) be satisfied, $f \in X_{1}$ and let $g, h: X_{2} \rightarrow X_{1}$ both satisfy (H4). If $\left(X_{1}, X_{2}\right)$ is admissible w.r.t. both $R$ and $I-R$ then for each sufficiently small $\epsilon>0$ there exists $\eta>0$ such that if $\|f\|_{1} \leqq \eta$ then (7) has a solution $x \in X_{2}$ with $\|x\|_{2} \leqq \epsilon$. 


\section{BIBLIOGRAPHY}

1. J. L. Massera and J. J. Schäffer, Linear differential equations and function spaces, Pure and Appl. Math., vol. 21, Academic Press, New York, 1966. MR 35 \#3197.

2. C. Corduneanu, Problèmes globaux dans la théorie des équations intégrals de Volterra, Ann. Mat. Pura Appl. (4) 67 (1965), 349-363. MR 32 \#331.

3. - Some perturbation problems in the theory of integral equations, Math. Systems Theory 1 (1967), 143-155. MR 35 \#4773.

4. H. A. Antosiewicz, Un analogue du principle du point fixe de Banach, Ann. Mat. Pura Appl. (4) 74 (1966), 61-64. MR 34 \#4962.

5. R. K. Miller, On the linearization of Volterra integral equations, J. Math. Anal. Appl. 23 (1968), 198-208. MR 37 \$5635.

6. R. K. Miller, J. A. Nohel and J. S. W. Wong, Perturbations of integral equations, J. Math. Anal. Appl. 24 (1969), 676-691.

7. I. W. Sandberg, On the $\mathcal{L}_{2}$-boundedness of solutions of nonlinear functional equations, Bell System Tech. J. 43 (1964), 1581-1599. MR 30 \#1416.

8. G. Zames, On the input-output stability of time-varying nonlinear feedback systems. I, II, IEEE Trans. Automatic Control AC-11 (1966), 228-238; 465-476.

9. R. E. A. C. Paley and N. Wiener, Fourier transforms in the complex domain, Amer. Math. Soc. Colloq. Publ., vol. 19, Amer. Math. Soc., Providence, R. I., 1934; reprint, 1967.

Broivn University, Providence, Rhode Island 02912 